\title{
IDENTIFICACIÓN DE HIDROCARBUROS AROMÁTICOS POLICÍCLICOS (HAPS) EN EL PM2.5 DEL AIRE DE PAMPLONA-COLOMBIA
}

\section{IDENTIFICATION OF POLYCYCLIC AROMATIC HYDROCARBONS (PAHS) IN THE AIR PM 2.5 PAMPLONA- COLOMBIA}

\begin{abstract}
Mónica Juliana Quijano ${ }^{1}$, Alfonso Quijano ${ }^{2}$, Iván Meléndez ${ }^{3}$
${ }^{1}$ Químico, Especialista en Bioquímica. Universidad de Pamplona. Investigadora Grupo de Investigación en Química, Pamplona, Colombnia. e-mail: mojuquiva@unipamplona.edu.co; ${ }^{2}$ Químico, Ph.D. Universidad de Pamplona. Profesor Asociado. Departamento de Química y Biología. Facultad de Ciencias Básicas. Grupo de Investigación en Química, e-mail: alfonsoquijanoparra@gmail.com; ${ }^{3}$ Biologo, M.Sc. Universidad de Pamplona. Profesor asistente. Departamento de Química y Biología. Facultad de Ciencias Básicas. Grupo de Investigación en Biología Molecular (Biomogen), e-mail: imgelvez@unipamplona. edu.co.com. Correspondencia: Ciudadela universitaria, Km 1, Vía Bucaramanga, Pamplona, Norte de Santander, Colombia. (Grupo de Investigación en Química).Teléfono: 097-5685303 ext: 215. Celular: 3166949582 e-mail: alfonsoquijanoparra@ unipamplona.edu.co
\end{abstract}

Rev. U.D.C.A Act. E Div. Cient. 17(1): 25-33, Enero-Junio, 2014

\section{RESUMEN}

La contaminación del aire, se considera un serio problema ambiental, debido a la presencia en la atmósfera de materiales peligrosos, tales como metales e hidrocarburos aromáticos policíclicos (HAPs) presentes en el material particulado (PM). Los (HAPs) son contaminantes prioritarios del aire, por sus propiedades extremadamente peligrosas para la salud humana. El material particulado fracción respirable PM2.5 fue monitoreado usando un equipo Partisol 2025 Plus con filtros de cuarzo Palmflex. Los filtros, se sometieron a extracción por ultrasonido, utilizando por separado tres solventes diclorometano, acetona y una mezcla diclorometano:acetona (3:1). Para identificar los HAPs presentes, los extractos obtenidos se analizaron en un cromatografo de gases HP6890 plus con detector FID. Por primera vez en el PM2.5 del aire de Pamplona-Norte de Santander, se detectaron 12 HPAs, dentro, de los cuales, se destaca el benzo [a] pireno, considerado uno de los más potentes carcinógenos en humanos.

Palabras clave: Material particulado, PM2.5, mutágenos, benzo(a)pireno, cromatografía de gases.

\section{SUMMARY}

Air pollution is considered a serious environmental problem due to the presence in the atmosphere of hazardous materials such as metals and polycyclic aromatic hydrocarbons (HAPs) content in the particulate matter (PM). The (PAHs) are priority pollutants from the air due to its extremely dangerous to human health. In order to identify the polycyclic aromatic hydrocarbons present in the organic matter from the air $\mathrm{PM}_{2.5}$ Pamplona, the respirable fraction $\mathrm{PM}_{2.5}$ particulate material was monitored using equipment Partisol 2025 Plus Palmflex quartz filters. The filters were subjected to ultrasonic extraction using dichloromethane three separate solvents, acetone and dichloromethane: acetone (3:1). To identify PAHs, the extracts were analyzed in a gas chromatograph with FID detector plus HP6890. For the first time in the air PM2.5 Pamplona-Norte de Santander, are detected and identified 12 PAHs, among which stands out the benzo [a] pyrene, which is considered one of the most potent carcinogens.

Key words: Particulate matter, PM2.5, mutagens, Benzo (a) pyrene, gas chromatography. 


\section{INTRODUCCIÓN}

Los tóxicos del aire son contaminantes y son conocidos o sospechosos de causar efectos adversos a la salud y se clasifican de la siguiente manera: contaminantes gaseosos, categoría a la cual pertenecen el Ozono ( $\left.0_{3}\right)$, el dióxido de azufre $\left(\mathrm{SO}_{2}\right)$, los óxidos de nitrógeno (NOx) y el monóxido de carbono (CO); compuestos Orgánicos Volátiles; contaminantes orgánicos persistentes; metales pesados y material particulado $\mathrm{PM}_{10}$ y $\mathrm{PM}_{2.5}$. En los humanos, la inhalación es la ruta más frecuente de acceso de los contaminantes atmosféricos al organismo, es por esto que el tracto respiratorio y los pulmones están generalmente involucrados en los procesos de translocación del agente nocivo, por la sangre y los tejidos (Halatek et al. 2005; Vinitketkumnuen et al. 2002). La contaminación del aire por material particulado (PM), se considera un serio problema ambiental, debido a la presencia en la atmósfera de materiales peligrosos, tales como metales traza, que aumentan las lesiones cardiopulmonares en los seres humanos (Shaheen et al. 2005; Carreras et al. 2013; Shah et al. 2006).

En cuanto a los efectos sobre la salud humana, especialmente enfermedades respiratorias, el material particulado fracción respirable es el de mayor preocupación, ya que la gente pasa cerca del 20\% de su tiempo al aire libre (Mannino \& Orecchio, 2008); a largo plazo, la exposición al PM se ha asociado con una mayor incidencia del aumento de la tasa de cáncer de pulmón (Goldberg et al. 2001) y enfermedades cardiovasculares (Dagouassat et al. 2012).

Estudios relacionados con las fuentes de emisión de las partículas finas ( $\mathrm{PM}_{2.5}$ ) comprueban que los vehículos que funcionan con diesel y gasolina son una de las principales fuentes de emisión de material particulado (Maykut et al. 2003; (U.S. EPA, 2004). El daño inducido por el PM, se produce por estrés oxidativo, a través de las especies reactivas de oxigeno (ROS), que se relacionan con procesos carcinogénicos (Milaeva, 2011; Hou et al. 2011; Zhang et al. 2001). Las partículas $\mathrm{PM}_{10}$ y $\mathrm{PM}_{2.5}$ muestran mutagenicidad directa e indirecta, atribuida principalmente a los hidrocarburos aromáticos policíclicos (HAPs) encontrados en las emisiones de vehículos diésel, especialmente (Amador-Muñoz et al. 2010). Estos (HAPs) son considerados como las principales causas de acción indirecta de mutagenicidad; muchos de los HAP individuales son citotóxicos, mutagénicos y potencialmente carcinógeno para los seres humanos. En algunos casos, la actividad carcinogénica es particularmente elevada, como es el ejemplo del benzo[a]antraceno en animales y el benzo[a] pireno en humanos (Hayakawa, 2009). La mutagenicidad de acció12n directa se le atribuye a los hidrocarburos aromáticos nitropoliciclicos (NPAHs), como los dinitropirenos (DNP) (Wada et al. 2001). Uno de los NPAHs más importantes es la 3-nitrobenzanthrona (3-nitro-7H-benzo [de] anthracen7-ona; 3-NBA), que es un mutágeno extremadamente potente y un carcinógeno en humanos, detectado en los gases de escape de motores Diésel (Da Costa et al. 2009; Wada et al. 2001). De acuerdo con la Agencia Internacional de Investigación contra el Cáncer, algunos son posiblemente carcinógenos para los humanos (Kawanaka et al. 2008); por ejemplo, el 1-nitropireno es uno de los compuestos con más poder mutagénico, al igual que algunos dinitro-PAHs (PortetKoltalo et al. 2008). Estudios epidemiológicos proporcionan suficiente evidencia de la carcinogenicidad en los seres humanos de las emisiones del diésel (U.S. EPA., 2002; Riger et al. 2011; Taga et al. 2005).

El interés en conocer los niveles ambientales de los HAPs radica fundamentalmente, en las propiedades carcinogénicas, mutagénicas y teratogénicas que poseen algunas de ellas, lo que los llevó a ser considerados como contaminantes prioritarios por la Agencia Norteamericana de Protección del Medioambiente (US-EPA) y la IARC (IARC, 2010).

En Colombia, se ha estudiado el PM 10 y se han identificado los HAPs presentes en esta fracción, es así como Pachón et al. (2004), en el estudio realizado en el aire de la zona metropolitana de Bogotá, hallaron fenantreno, antraceno, fluoranteno, pireno, benzo(a)antraceno, criseno, benzo(ghi) perileno e Indeno(1,2,3-cd)pireno. En la investigación realizada en las partículas respirables de áreas industriales y de áreas afectadas por el tráfico vehicular de Bogotá, se determinó la presencia de benzo(a)pireno, sustancia cancerígena y asociada a las emisiones de vehículos y combustión del carbón (Consuegra, 2006).

En Colombia, no existen normas para los HAPs; la deficiencia de estudios y en parte el desconocimiento de la existencia de estas sustancias (HAPs, NHAPs, OHAPs) en el material particulado $P M_{2.5}$, han incidido en la falta de acciones que prevengan, reduzcan, minimicen, mitiguen y controlen la emisión de estas sustancias a la atmósfera. Por lo tanto, investigaciones como ésta, se enfocan en mostrar el impacto que pueden tener los HAPs en la salud humana, para que, de esa manera, se puedan tomar medidas de prevención, que se podrían reflejar en el desarrollo de nuevas investigaciones, así como en la implementación de políticas de control para darle cumplimiento al numeral 6 del artículo 1 de la Ley 99 de 1993, "Principios Generales Ambientales".

Teniendo en cuenta que a Pamplona la atraviesa una vía nacional que presenta un alto flujo vehicular, especialmente, de vehículos de carga pesada que funcionan con diésel, en el presente estudio pretendemos identificar compuestos mutagénicos (HAPs), originados por la combustión del diésel. Es de anotar que esta ciudad no tiene industrias que emitan 
contaminantes a la atmosfera, por lo que la contaminación ambiental en esta zona del país proviene, fundamentalmente, de la combustión de los vehículos a diésel y gasolina.

\section{MATERIALES Y MÉTODOS}

Sitio de muestreo: Se realizó el monitoreo de la fracción respirable $\mathrm{PM}_{2.5}$ en Pamplona, Norte de Santander, capital ubicada en la cordillera Oriental de Colombia, con coordenadas geográficas $72^{\circ} 25$ de longitud Oeste y $7^{\circ} 20$ de latitud Norte, a una altitud de 2100 msnm y una presión atmosférica de $542 \mathrm{~mm}$ de $\mathrm{Hg}$. EL monitoreo del material particulado ultrafino PM2.5 se realizó con un equipo Partisol-2025 PLUS de la Ruprecht-Patashnick; el equipo se instaló en la azotea del edificio de la facultad de Ciencias Básicas de la Universidad de Pamplona, cerca de una vía nacional transitada por vehículos que funcionan con diésel y gasolina. Las muestras ambientales obtenidas en muestreos de 24 horas, cada tres días, se realizaron durante el período comprendido entre enero-julio del 2011.Para la recolección, se utilizaron filtros de Teflón de $47 \mathrm{~mm}$ de diámetro, con un tamaño de poro de 2 micras.

Extracción de la Materia Orgánica de los filtros de PM2.5: La materia orgánica (MO) de los filtros de PM2.5 (HAPs), se extrae por ultrasonido, en un baño ultrasónico (Branson 1510); como solventes de extracción, se utilizan, por separado, el diclorometano $(200 \mathrm{~mL})$, la acetona $(200 \mathrm{~mL})$ y una mezcla de acetona: diclorometano (1:3). Los filtros de PM2.5, inicialmente, se introducen en un vaso de precipitado con $20 \mathrm{~mL}$ del solvente, para un periodo de extracción de 15 minutos, a una temperatura de $23^{\circ} \mathrm{C}-24^{\circ} \mathrm{C}$; se recoge el extracto y se agregan de nuevo $20 \mathrm{~mL}$ del solvente y se realiza la extracción durante 15 minutos; se recoge el extracto y se repite la operación hasta completar los $200 \mathrm{~mL}$ del solvente. De esta manera, se obtienen tres extractos por separado, para cada uno de los solventes utilizados.

Concentración de la materia orgánica: Una vez obtenido el extracto $(200 \mathrm{~mL})$, se concentra en un evaporador rotatorio de vacío, marca Heidolph modelo Laborota 400-1, a la temperatura de ebullición del solvente a 150rpm, hasta aproximadamente $2 \mathrm{~mL}$ obteniéndose el extracto global. Posteriormente, los extractos globales, se utilizan para la determinación de HAPs por Cromatografía de Gases (Ping $\varepsilon$ Panuwat, 2006). Las muestras, se secaron con $\mathrm{Na}_{2} \mathrm{SO}_{4}$, con el fin de eliminar el agua residual y preparar la muestra para el análisis cromatográfico. Se guardaron en frasco ámbar, manteniéndolas refrigeradas a $4^{\circ} \mathrm{C}$.

Identificación de Hidrocarburos Aromáticos Poli cíclicos (HAPs): Para identificar los HAPs extraídos con Diclorometano, Acetona y la mezcla Acetona:Diclorometano, se utilizó un equipo de Cromatografía de Gases marca Agilent Te- chnologies 6890A Plus Series II Hewlet-Packard Plus, con detector FID (Flame Ionization Detector). La columna utilizada es Agilent DB-EUPAH 20m de longitud, 0,18mm de diámetro, $0,25 \mu \mathrm{m}$ de diámetro interno ( $5 \%$ phenyl methylpolysiloxane). Para la identificación de los HAPs, se empleó el patrón de 16 hidrocarburos de Restek (catalogo \# 31841 EPA Method 8310 PAH Mixture). La identificación cualitativa de los HAPs presentes en el extracto global, se realizó de acuerdo a las siguientes condiciones: detector FID, a $320^{\circ} \mathrm{C}$ Mezcla (mL/min): Aire $400-\mathrm{H}_{2} 30-\mathrm{N}_{2} 45$. Se inyectó $1 \mu \mathrm{l}$, modo splitless, a $320^{\circ} \mathrm{C}$. Temperatura inicial de $45^{\circ} \mathrm{C}$, por 0,8 min y se incrementó de la siguiente manera: $45^{\circ} \mathrm{C} / \mathrm{min}$ hasta $200^{\circ} \mathrm{C}, 2,50^{\circ} \mathrm{C} / \mathrm{min}$ hasta $225^{\circ} \mathrm{C}, 3^{\circ} \mathrm{C} / \mathrm{min}$ hasta $266^{\circ} \mathrm{C}, 5^{\circ} \mathrm{C} /$ min hasta $300^{\circ} \mathrm{C}, 10^{\circ} \mathrm{C} / \mathrm{min}$ hasta $320^{\circ} \mathrm{C}$ durante $4,50 \mathrm{~min}$. Tiempo de análisis por muestra 41.21min. Gas de arrastre Helio, flujo $20 \mathrm{~mL} / \mathrm{min}$. Temperatura del inyector $250^{\circ} \mathrm{C}$.

\section{RESULTADOS Y DISCUSIÓN}

Para realizar la identificación de los HAPs, se tomó como referencia el cromatograma de la muestra patrón de $16 \mathrm{Hi}$ drocarburos aromáticos poli cíclicos que la US-EPA incluye en su lista de contaminantes prioritarios (EPA, 1984) y que pueden estar presentes en los extractos globales del $\mathrm{PM}_{2.5}$ de Pamplona (Figura 1).

Del extracto global extraído con acetona, se obtuvo el perfil cromatográfico en el que se observa compuestos a los 11, 15, 21, 24 y 38 minutos, los cuales, corresponden al benzo[c] fluoreno, benzo[a]antraceno, benzo[b]fluoranteno, benzo[a]pireno, dibenzo[a,h]pireno, mostrados en la figura 2. En el perfil cromatográfico del extracto global extraído con la mezcla acetona:diclorometano (1:3) podemos observar compuestos a los 11, 16, 21, 22 y 24 minutos, que corresponden a los compuestos benzo[c]fluoreno, Criseno, benzo[b] fluranteno, benzo[k]fluranteno y benzo[a] pireno (Figura 3). El perfil cromatográfico del extracto global extraído con diclorometano, se muestra en la figura 4. En dicha figura, se observan compuestos a los 11, 13, 16, 21, 24, 31, $31,5,32,37$ y 40 minutos, que corresponden a los compuestos benzo[c]fluoreno, benzo[a]antraceno, 5 metil criseno, benzo[j]fluoranteno, benzo[a]pireno, Indeno[1,2,3-cd]pireno dibenzo[a,h]antraceno, benzo(g,h,i)perileno, dibenzo[a,e]pireno, dibenzo[a,h] pireno. Es necesario señalar, que el benzo $[g, h, i]$ perileno es un indicador de HAPs emitidos por los escapes de los motores diésel y de gasolina, como lo reporta Kuo et al. (1998). Estos hallazgos son importantes, debido a que se ha establecido que uno de los factores de riesgo para la mortalidad por cáncer es el tráfico vehicular (Anand et al. 2008; Belpomme et al. 2007). Además, el Programa Nacional de Toxicología Americano (NTP) anticipa que contaminantes, como el benzo[a] antraceno, benzo[b] fluoranteno, benzo[j] fluoranteno, benzo[k] fluoranteno, benzo[a] pireno, dibenzo[a,h] antraceno, dibenzo[a,e] pireno, dibenzo[a,i] pi- 


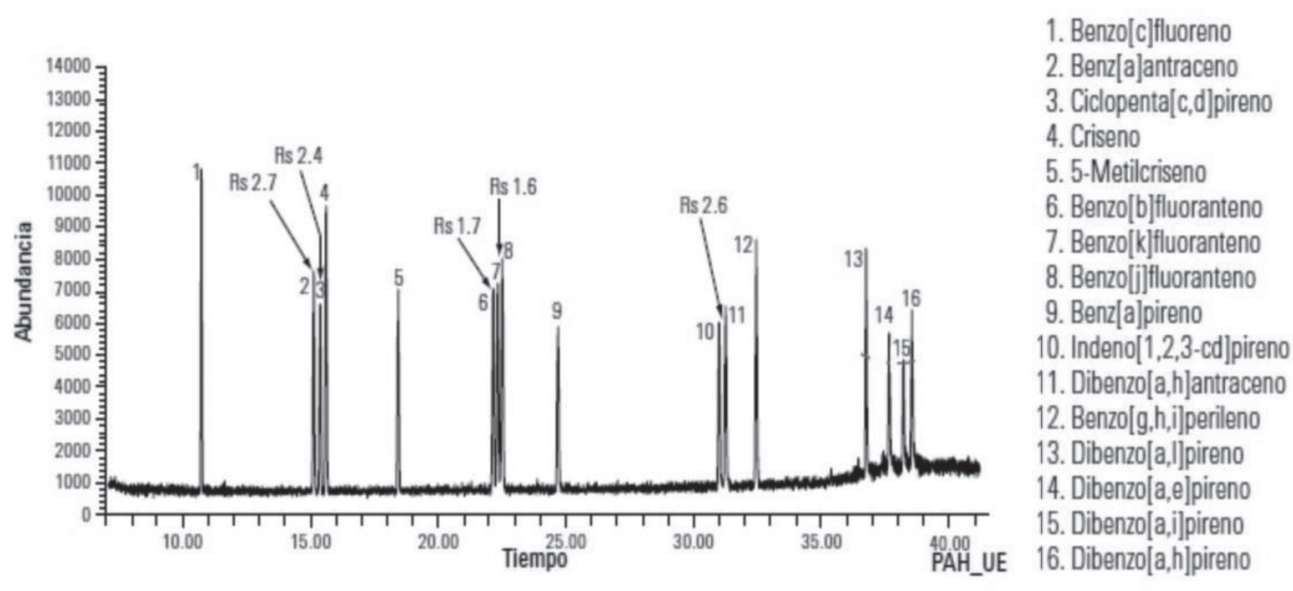

Figura 1. Cromatograma de la muestra patrón de 16 Hidrocarburos (Catálogo \# 31841 EPA Method 8310 PAH Mixture).

reno, dibenzo[a,l] pireno, indeno[1,2,3-c,d] pireno y 5-metilcriseno, pueden ser considerados como carcinógenos en humanos (RoC, 2009).
Como se observa en la tabla 1 , al utilizar la acetona como solvente de extracción, permite identificar 5 HAPs; el diclorometano permite identificar 10 HAPs y la mezcla dicloro-

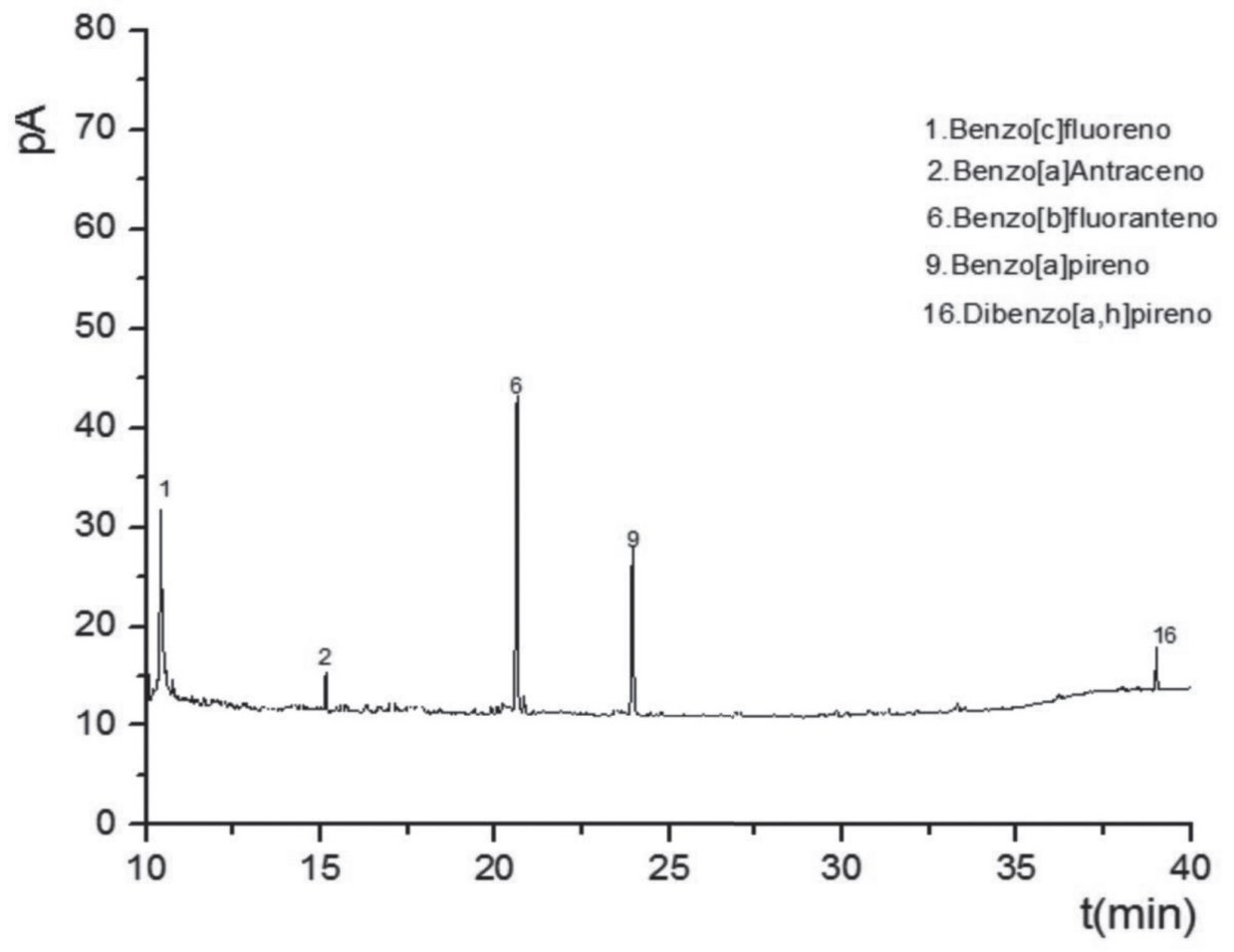

Figura 2. Perfil cromatográfico del extracto global de los HAPs presentes en el PM2.5 de la ciudad de Pamplona, utilizando como solvente de extracción acetona. 


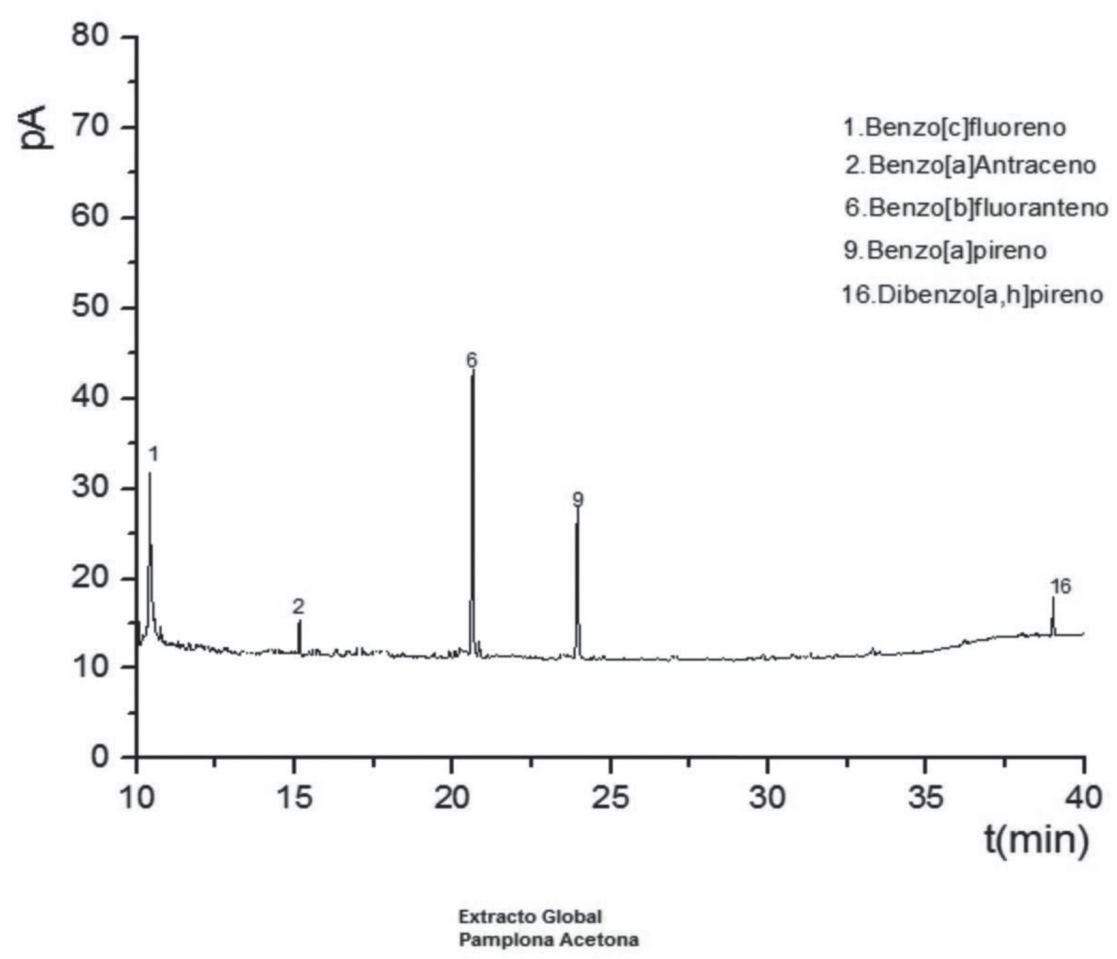

Figura 3. Perfil cromatográfico de los HAPs presentes en el PM2.5 de la ciudad de Pamplona, extraídos con una mezcla de acetona:diclorometano(1:3).

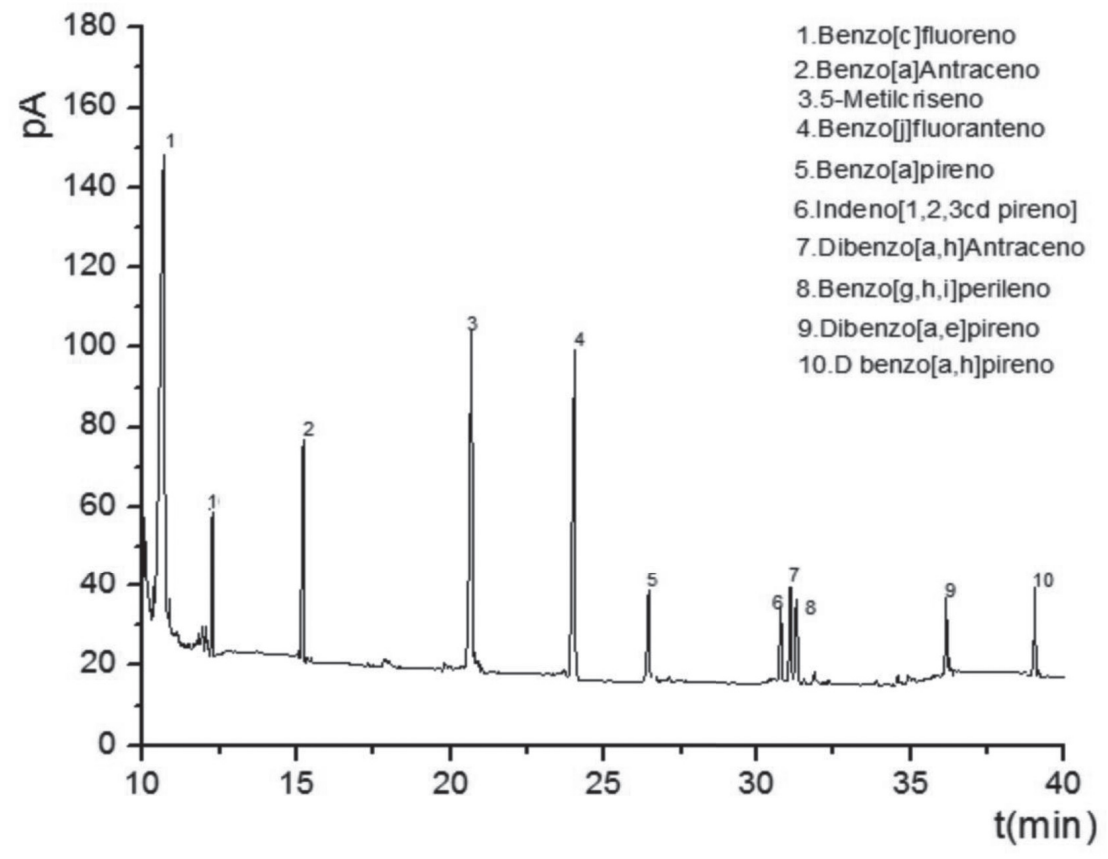

Extracto Global

Pamplona DCM

Figura 4. Perfil cromatográfico de los HAPs presentes en el extracto global del PM2.5 del aire de Pamplona, extraído con diclorometano. 
Tabla 1. HAPs identificados en la materia orgánica del PM2.5 del aire de Pamplona, extraído con diferentes solventes.

\begin{tabular}{|l|c|c|c|}
\hline \multicolumn{1}{|c|}{ HAPs } & Acetona & DCM & Acetona: DCM(1:3) \\
\hline Benzo(fluoreno & $\mathrm{X}$ & $\mathrm{X}$ & $\mathrm{X}$ \\
\hline Benzo(a)antraceno & $\mathrm{X}$ & $\mathrm{X}$ & $\mathrm{X}$ \\
\hline Benzo(b)fluoranteno & $\mathrm{X}$ & $\mathrm{X}$ & $\mathrm{X}$ \\
\hline Benzo(a)pireno & $\mathrm{X}$ & $\mathrm{X}$ & $\mathrm{ND}$ \\
\hline Dibenzo(a,h)pireno & $\mathrm{X}$ & $\mathrm{ND}$ & $\mathrm{X}$ \\
\hline Criseno & $\mathrm{ND}$ & $\mathrm{ND}$ & $\mathrm{ND}$ \\
\hline Benzo(k)fluoranteno & $\mathrm{ND}$ & $\mathrm{X}$ & $\mathrm{ND}$ \\
\hline 5 Metil criseno & $\mathrm{ND}$ & $\mathrm{X}$ & $\mathrm{ND}$ \\
\hline Benzo(j)fluoranteno & $\mathrm{ND}$ & $\mathrm{X}$ & $\mathrm{ND}$ \\
\hline Indeno(1,2,3,cd)pireno & $\mathrm{ND}$ & $\mathrm{X}$ & $\mathrm{ND}$ \\
\hline Dibenzo(a,h)antraceno & ND & $\mathrm{X}$ & $\mathrm{ND}$ \\
\hline Benzo(g,h,i)perileno & ND & $\mathrm{X}$ & \\
\hline Dibenzo(a,h)pireno & ND & & \\
\hline
\end{tabular}

$\mathrm{X}$ identificado; ND no detectado.

metano acetona 5 HAPs. Estos tres solventes identifican en común al Benzo(a)pireno, considerado carcinógeno en humanos (Straif et al. 2006; Ducatti \& Vargas, 2003). De los 10 HAPs identificados con diclorometano, 6 son considerados como posibles o probables carcinógenos en humanos (IARC, 2010); es de resaltar que estos 6 HAPs no se extraen con acetona ni con la mezcla acetona:diclorometano. Es necesario realzar la importancia del uso de mezclas como solventes de extracción de la materia orgánica del material particulado, ya que en nuestra investigación, el uso de esta mezcla permitió identificar dos compuestos no detectados cuando se usó diclorometano o acetona. Estos compuestos, el criseno y el benzo(k)fluoranteno son catalogados como posibles o probables carcinógenos en humanos. En nuestra investigación observamos que el solvente más eficiente para la identificación de los HAPs es el diclorometano, solvente recomendado por diferentes investigadores (Hanzalova et al. 2010; Vargas, 2003; Claxton et al. 2001), para extraer la materia orgánica presente en el material particulado.

Como se mencionó anteriormente, por la ciudad de Pamplona circula un tráfico pesado de vehículos, cuyos motores funcionan con diésel, por lo que es probable que en el aire de Pamplona estén presentes compuestos nitro-HAPs, que corroboran la apreciación encontrada en el estudio de Meléndez Gélvez et al. (2012), en el cual, se encontró que el material particulado ( $P M_{2.5}$ ), presente en las muestras de aire de Pamplona, es potencialmente mutagénico y genotoxico, efectos que son atribuibles a los metales e HAPs, coincidien- do con los resultados obtenidos por Da Costa et al. (2009), quienes reportan que los contribuyentes más importantes a la mutagenicidad ambiental son naftaleno 1,2 metil naftaleno, fluoreno, dibenzotiofeno, fenantreno, fluoranteno y pireno.

Del presente estudio, se concluye que de los 13 HPAs identificados en el aire de la ciudad de Pamplona, la mayoría representa un riesgo para la salud de la población, porque se conoce de la estrecha relación que existe entre la exposición a estos compuestos y la aparición de enfermedades, especialmente la generación de diferentes tipos de cáncer.

Este estudio evidencia la asociación entre la calidad del aire de la ciudad de Pamplona (PM2.5) y la exposición a sustancias contaminantes producidas por la combustión de las fuentes móviles que allí circulan.

Se evidencia la importancia del uso de diferentes solventes en la extracción de la materia orgánica del PM2.5, lo que permite una mayor información para la identificación de los HAPs.

Agradecimientos: A la Rectoría de la Universidad de Pamplona, por su apoyo financiero a este proyecto y a la colaboración prestada por el personal adscrito al Laboratorio de Control de Calidad de la Universidad de Pamplona. Conflicto de intereses: El manuscrito fue preparado y revisado con la participación de todos los autores, quienes declaramos que no existe ningún conflicto de intereses que ponga en riesgo 
la validez de los resultados presentados. Financiación: Este estudio fue financiado por la Rectoría de la Universidad de Pamplona y el Grupo de Investigación en Química de la Universidad de Pamplona.

\section{BIBLIOGRAFÍA}

1. AMADOR-MUÑOZ, O.; VILLALOBOS-PIETRINI, R.; AGAPITO-NADALES, M.C.; MUNIVE-COLIN, Z.; HERNANDEZ-MENA, L.; SÁNCHEZ-SANDOVAL, M. 2010. Solvent extracted organic matter and polycyclic aromatic hydrocarbons distributed in size-segregated airborne particles in a zone of México city: seasonal behavior and human exposure. Atmosph. Environ. 44:122-130.

2. ANAND, P.; KUNNUMAKARA, A.; SUNDARAM, C.; HARIKUMAR, K.; THARAKAN, S.; LAI, O.J.; SUNG, B.; AGGARWAL, B.B. 2008. Cancer is a preventable disease that requires major lifestyle changes. Pharm. Res. 25(9):2097-116.

3. BELPOMME, D.; IRIGARAY, P.; HARDELL, L.; CLAPP, R.; MONTAGNIERE, L.; EPSTEIN, S.; SASCO, A.J. 2007. The multitude and diversity of environmental carcinogens. Environ. Res.105:414-29.

4. CONSUEGRA, A.A. 2006. Estudio de la contaminación atmosférica generada por óxidos de azufre, óxidos de Nitrógeno y material particulado en un sector de la ciudad de Bogotá. Primera parte. Avances investigación en Ingenieria.22:32-36.

5. CARRERAS H., A.; CALDERÓN-SEGURA M, E.; GÓMEZ-ARROYO, S.; MURILLO-TOVAR, M.; AMADORMUÑOZ, O. 2013. Composition and mutagenicity of PAHs associated with urban airborne particles in Córdoba, Argentina. Environ. Poll. 178:403-410.

6. CLAXTON, L.D.; WARREN, S.; ZWEIDINGER, R.; CREASON, J. 2001. A comparative assessment of Boise, Idaho, ambient air fine particle samples using the plate and microsuspension Salmonella mutagenicity assays. Sci. Total Environ. 275(1-3):95-108.

7. DA COSTA, G.; SINGH, R.; ARLT, V.M.; MIRZA, A.; RICHARDS, M.; TAKAMURA-ENYA, T.; SCHMEISER, H.H.; FARMER, P.B.; PHILLIPS, D.H. 2009. Quantification of 3-nitrobenzanthrone-DNA adducts using online column-switching HPLC-electrospray tandem mass spectrometry. Chem. Res. Toxicol. 22(1):18601868.
8. DAGOUASSAT, M.; LANONE, S.; BOCZKOWSKI, J. 2012. Interaction of matrix metalloproteinases with pulmonary pollutants. Eur. Respir. J. 39(4):10211032.

9. DUCATTI, A.; VARGAS, V.M.F. 2003. Mutagenic activity of airborne particulate matter as an indicative measure of atmospheric pollution. Mutat. Res. 540:6777.

10. EPA, 1984. Environmental Protection Agency. Health Effects Assessment for Polycyclic Aromatic Hydrocarbons (PAHs). EPA-540/1-86-013. Chicago.

11. GOLDBERG, M.S.; BURNETT, R.T.; BAILAR, J.C.; BROOK, J.; BONVALOT, Y.; TAMBLYN, R.; SINGH, R.; VALOIS, M.F.; VINCENT, R. 2001.The association between daily mortality and ambient air particle pollution in Montreal, Quebec. Environ. Res. 86:26-36.

12. HALATEK, T.; SINCZUK-WALCZAK, H.; SZYMCZAK, M.; RYDZYNSKI, K. 2005. Neurological and respiratory symptoms in shipyard welders exposed to manganese. J. Occup. Med. Environ. Health. 18(3):265-274.

13. HANZALOVA, K.; ROSSNER, P. Jr.; SRAM, R.J. 2010. Oxidative damage induced by carcinogenic polycyclic aromatic hydrocarbons and organic extracts from urban air particulate matter. Mutation Res. 696(2):114-121.

14. HAYAKAWA, K. 2009. Atmospheric Pollution and Its Countermeasure in East Asia from the Viewpoint of Polycyclic Aromatic Hydrocarbons. J. Health Sci. 55:870-878.

15. HOU, L.; ZHANG, X.; TARANTINI, L.; NORDIO, F.; BONZINI, M.; ANGELICI, L.; MARINELLI, B.;RIZZO, G.; CANTONE, L.; APOSTOLI, P.; BER TAZZI, P.A.; BACCARELLI, A. 2011. Ambient PM exposure and DNA methylation in tumor suppressor genes: a cross-sectional study. Part. Fibre. Toxicol. 30:8-25.

16. IARC. International Agency for Research on Cancer. 2010. Some nonheterocyclic polycyclic aromatic hydrocarbons and some related exposures. IARC Monographs on the Evaluation of Carcinogenic Risks to Humans, 92. p.765-773.

17. KAWANAKA, Y.; MATSUMOTO, E.; WANG, N.; YUN, S.J.; SAKAMOTO, K. 2008. Contribution of nitrated polycyclic aromatic hydrocarbons to the mutagenicity of ultrafine particles in the roadside atmosphere. Atmosph. Environ. 42:7423-7428. 
18. KUO, C.Y.; CHENG, T.W.; CHEN, C.Y.; LEE, H. 1998. Correlation between the amounts of polycyclic aromatic hydrocarbons and mutagenicity of airborne particulate samples from Taichung City. Taiwan. Environ. Res. 78:43-49.

19. MANNINO, M.R.; ORECCHIO, S. 2008. Polycyclic aromatic hydrocarbons (PAHs) in indoor dust matter of Palermo (Italy) area: Extraction, GC-MS analysis, distribution and sources. Atmos. Environ. 42:18011817.

20. MAYKUT, N.N.; LEWTAS, J.; KIM, E.; LARSON, T.V. 2003. Source Apportionment of PM2.5 at an Urban Improve site in Seattle, WA. Environ. Sci. Technol. 37:5135-5142.

21. MELÉNDEZ GÉLVEZ, I.; MARTÍNEZ MONTAÑEZ, M.L.; QUIJANO PARRA, A. 2012. Actividad mutagénica y genotóxica en el material particulado fracción respirable $M P_{2,5}$, en Pamplona, Norte de Santander, Colombia. Latreia. 25(4):347-356.

22. MILAEVA, E.R. 2011. Metal-based antioxidants--potential therapeutic candidates for prevention the oxidative stress-related carcinogenesis: mini-review. Curr. Top Med Chem. 11(21):2703-2713.

23. PACHÓN, J.; GARCÍA, H.; BUSTOS, M.; BRAVO, H.; SOSA, R. 2004. Determinación de hidrocarburos aromáticos policíclicos en muestras de partículas atmosféricas en la zona metropolitana de la ciudad de Bogotá (Colombia). Meteorol. Colomb. 8:27-35.

24. PING, L.; PANUWAT, H. 2006. Characterization of polycyclic aromatic hydrocarbons (PAHs) on lime spray dryer (LSD) ash using different extraction methods. Chemosph. 62:265-274.

25. PORTET-KOLTALO, F.; OUKEBDANE, K.; DIONNET, F.; DESBENE, P.L. 2008. Optimisation of the extraction of polycyclic aromatic hydrocarbons and their nitrated derivatives from diesel particulate matter using microwave-assisted extraction. Analyt. Bioanalyt. Chem. 390:389-398.

26. RIGER, C.J.; FERNANDES, P.N.; VILELA, L.F.; MIELNICZKI-PEREIRA, A.A.; BONATTO, D.; HENRIQUES, J.A.; ELEUTHERIO, E.C. 2011. Evaluation of heavy metal toxicity in eukaryotes using a simple functional assay. Metallomics. 3(12):1355-1361.

27. RoC, 2009. National Toxicology Program (NTP): Report on Carcinogens. U. S. Department of Health and Hu- man services, Public Health Service. National Toxicology Program (NTP). s.p.

28. SHAH, M.H.; SHAHEEN, N.; JAFFAR, M.; KHALIQUE, A.; TARIQ, S.R.; MANZOOR, S. 2006. Spatial variations in selected metal contents and particle size distribution in an urban and rural atmosphere of Islamabad, Pakistan. J. Environ. Manag. 78:128-137.

29. SHAHEEN, N.; SHAH, M.H.; KHALIQUE, A.; JAFFAR, M. 2005. Metal levels in airborne particulate matter in urban Islamabad, Pakistan. Bull. Environ. Contam. Tox. 75(4):739-746.

30. STRAIF, K.; BAAN, R.; GROSSE, Y.; SECRETAN, B.; EI, G.F.; COGLIANO, V. 2006. Carcinogenicity of household solid fuel combustion and high temperature frying, Lancet Oncol. 7:977-978.

31. TAGA, R.; TANG, N.; HATTORI, T.; TAMURA, K.; SAKAI, S.; TORIBA, A.; KIZU, R.; HAYAKAWA, V. 2005. Direct-acting mutagenicity of extracts of coal burningderived particulates and contribution of nitropolycyclic aromatic hydrocarbons. Mutat. Res. 581:91-95.

32. U.S. EPA. 2002. Health Assessment Document for Diesel Engine Exhaust, US Environmental Protection Agency, Office of Research and Development, National Center for Environmental Assessment, Washington, DC. (EPA/600/8-90/057F). p.2-79-2-85.

33. U.S. EPA. 2004. Air Quality Criteria for Particulate Matter, US Environmental Protection Agency, Office of Research and Development, National Center for Environmental Assessment, Research Triangle Park Office, Research Triangle Park, NC, 2004 (vol. I, EPA/600/P-99/002aF, and vol. II, EPA/600/P99/002bF). p.7-186-7-192.

34. VARGAS, V.M.F. 2003. Mutagenic activity as a parameter to assess ambient air quality for protection of the environment and human health. Mutat. Res. 544:313319.

35. VINITKETKUMNUEN, U.; KALAYANAMITRA, K.; CHEWONARIN, T.; KAMENS, R. 2002. Particulate matter, PM10 \& PM2.5 levels, and airborne mutagenicity in Chiang Mai, Thailand. Mutat. Res. 519:121131.

36. WADA, M.; KIDO, H.; KISHIKAWA, N.; TOU, T.; TANAKA, M.; TSUBOKURA, J.; SHIRONITA, M.; MATSUI, M.; KURODA, N.; NAKASHIMA, K. 2001. Assessment of air pollution in Nagasaki city: determination of 
polycyclic aromatic hydrocarbons and their nitrated derivatives, and some metals. Environm. Pollut. 115:139-147.
37. ZHANG, W.; CUI, J.; Qi, Q. 2001. Advances on the biological effect indices for fine particles (PM2.5) in air. Wei Sheng Yan Jiu. 30(6):379-82.

Recibido: Agosto 26 de 2013

Aceptado: Diciembre 9 de 2013

Como citar:

Quijano, M.J.; Quijano, A.; Meléndez, I. 2014. Identificación de hidrocarburos aromáticos policíclicos (HAPS) en el PM2.5 del aire de Pamplona-Colombia. Rev. U.D.C.A Act. \& Div. Cient. 17(1): 25-33. 
